\title{
Índice HOMA-IR como indicador de riesgo de enfermedades endocrino-metabólicas en niños y adolescentes con obesidad
}

\author{
HOMA-IR Index as indicator of the risk of Endocrine-Metabolic diseases in obese \\ children and adolescents
}

\section{Índice HOMA-IR como indicador do risco de doenças metabólicas endócrinas em crianças e adolescentes com obesidade}

\author{
Jessica Valverde Pulla \\ veroval1991@gmail.com \\ https://orcid.org/0000-0003-3467-9237
}

Carem Prieto Fuentemayor

carem.prieto@ucacue.edu.ec

https://orcid.org/0000-0002-7752-932X

\section{Programa de Maestría en Diagnostico de Laboratorio Clínico y Molecular, Universidad Católica de Cuenca-Ecuador}

Laboratorio Clínico Espíritu Santo, Cuenca-Ecuador

Recibido 19 de marzo 2021 | Arbitrado y aceptado 13 de abril 2021 | Publicado en 4 de mayo 2021

\begin{abstract}
RESUMEN
La obesidad infantil contribuye a la presencia de Resistencia a la Insulina (IR), lo que a su vez incrementa el riesgo de padecer alteraciones endocrino-metabólicas en la niñez. El HOMA-IR ha demostrado tener gran utilidad para la detección y seguimiento de estas patologías, sin embargo, se deben considerar las variables fisiológicas y patológicas a las que está sujeto su cálculo. Además tiene una buena correlación con el clamp euglucémico para la detección de IR, pero es indispensable marcar puntos de corte específicos para diagnosticar e identificar a los pacientes pediátricos con alto riesgo de Síndrome Metabólico y Diabetes 2, lo que evitaría las complicaciones en la adultez. Objetivo. Es evaluar el HOMAIR, como indicador de riesgo de enfermedades endocrino-metabólicas en la población pediátrica con obesidad; así como mostrar sus limitaciones y utilidad diagnóstica. Método. Se realizó una revisión sistemática en las bases: Science Direct, Scopus, Scielo y Pubmed; utilizando los descriptores: "Índice HOMA-IR", "Obesidad Infantil" y "Resistencia a la Insulina". La búsqueda se restringió a artículos completos en español, inglés y portugués, entre 2007 y 2020. Se identificaron 3855 artículos, luego de aplicar las fases de análisis, se seleccionaron 10 artículos para la revisión, cada uno con puntos de corte específicos para la población estudiada y su respectiva curva ROC. Conclusión. Se concluyó que el HOMAIR es un modelo confiable para el diagnóstico de IR temprana en niños, lo que permite una intervención terapéutica preventiva y diagnóstica, es un excelente predictor de enfermedades endocrino-metabólicas y complicaciones cardiovasculares futuras.
\end{abstract}

Palabras clave: HOMA-IR; Resistencia a la insulina; obesidad pediátrica; enfermedades endocrinometabólicas

\begin{abstract}
Childhood obesity contributes to the presence of Insulin Resistance (IR), which in turn increases the risk of endocrine-metabolic disorders in childhood. The HOMA-IR has proven to be very useful for the detection and monitoring of these pathologies, however, the physiological and pathological variables to which its calculation is subject must be considered. It also has a good correlation with the euglycemic clamp for the detection of IR, but it is essential to mark specific cut-off points to diagnose and identify pediatric patients at high risk of Metabolic Syndrome and Diabetes 2, which would avoid complications in adulthood. Objective. It is to evaluate the HOMA-IR, as an indicator of risk of endocrine-metabolic diseases in the pediatric population with obesity; as well as showing its limitations and diagnostic utility. Method. A systematic review was carried out in the bases: Science Direct, Scopus, Scielo and Pubmed; using the descriptors: "HOMA-IR Index", "Childhood Obesity" and "Insulin Resistance". The search was restricted to complete articles in Spanish, English and Portuguese, between 2007 and 2020. 3855 articles were identified, after applying the analysis phases, 10 articles were selected for the review, each with specific cut-off points for the population. studied and its respective ROC curve. Conclution. It was concluded that the HOMA-IR is a reliable model for the diagnosis of early IR in children, which allows a preventive and diagnostic therapeutic intervention, it is an excellent predictor of endocrine-metabolic diseases and future cardiovascular complications.
\end{abstract}

JV: Licenciada en Laboratorio Clínico, Universidad de Cuenca. Laborando en el laboratorio clínico del Centro Medico privado "Espíritu Santo" de la parroquia Baños de la ciudad de Cuenca. Universidad Católica de Cuenca, Ecuador.

CP: Licenciada en Bioanálisis. Magister Scientiarum en Metabolismo Humano. Doctora en Ciencias de la Salud. Docente universitaria en el área de biología celular y molecular y bioquímica, con amplia experiencia en investigación en biología molecular. Universidad Católica de Cuenca, Ecuador.

Key words: : HOMA-IR; insulin resistance; pediatric obesity; endocrine-metabolic diseases 
JV: Licenciada en Laboratorio Clínico, Universidad de Cuenca. Laborando en el laboratorio clínico del Centro Medico privado "Espíritu Santo" de la parroquia Baños de la ciudad de Cuenca. Universidad Católica de Cuenca, Ecuador.

CP: Licenciada en Bioanálisis. Magister Scientiarum en Metabolismo Humano. Doctora en Ciencias de la Salud. Docente universitaria en el área de biología celular y molecular y bioquímica, con amplia experiencia en investigación en biología molecular. Universidad cólica de

\section{RESUMO}

A obesidade infantil contribui para a presença de Insulin Resistance (IR), que por sua vez aumenta o risco de distúrbios endócrino-metabólicos na infância. O HOMA-IR provou ser muito útil para a detecção e monitoramento destas patologias; entretanto, as variáveis fisiológicas e patológicas às quais seu cálculo está sujeito devem ser levadas em conta. Ela também tem uma boa correlação com a pinça euglycaemic para a detecção de IR, mas é essencial estabelecer pontos de corte específicos para diagnosticar e identificar pacientes pediátricos com alto risco de Síndrome Metabólica e Diabetes 2, o que evitaria complicações na vida adulta. Objetivo. Avaliar o HOMA-IR como um indicador de risco de doenças endócrino-metabólicas na população pediátrica com obesidade, bem como mostrar suas limitações e utilidade diagnóstica. Método. Uma revisão sistemática foi realizada nas seguintes bases de dados: Science Direct, Scopus, Scielo e Pubmed; utilizando os descritores: "HOMA-IR Index", "Childhood Obesity" e "Insulin Resistance". A busca foi restrita a artigos completos em espanhol, inglês e português, entre 2007 e 2020. Um total de 3855 artigos foram identificados, e após a aplicação das fases de análise, 10 artigos foram selecionados para a revisão, cada um com pontos de corte específicos para a população estudada e sua respectiva curva ROC. Conclusão. Concluiu-se que o HOMA-IR é um modelo confiável para o diagnóstico de IR precoce em crianças, permitindo intervenção terapêutica preventiva e diagnóstica, e é um excelente preditor de doenças endocrinometabólicas e futuras complicações cardiovasculares.

Palavras-chave: HOMA-IR; resistência à insulina; obesidade pediátrica; doenças endócrino-metabólicas

\section{INTRODUCCIÓN}

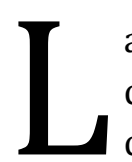
a Obesidad Infantil

(OI) es considerada una enfermedad crónica, sistémica y multifactorial; en la que intervienen tanto factores genéticos como del estilo de vida y entorno. Según datos de la Organización Mundial de la Salud (OMS), en 2016 la prevalencia de esta patología fue de 18\% en niños y adolescentes (de 5 a 19 años) (1). Debido a que su incidencia va en aumento y se mantiene hasta la adultez, constituye un importante problema en la salud pública (2).

La OI es la alteración nutricional infanto-juvenil más frecuente en pediatría, contribuye a la presencia de enfermedades crónicas no transmisibles como: Resistencia a la insulina (IR), Síndrome Metabólico (SM), Diabetes Mellitus 2 (DM2) e hipertensión arterial (3). Estas patologías incrementan el riesgo de padecer alteraciones cardiovasculares en la edad adulta $(3,4)$. En la actualidad se ha planteado como una meta a nivel mundial, controlar la incidencia de obesidad, haciendo énfasis en los niños y adolescentes. A pesar, que estas enfermedades no son causa de mortalidad alta en pediatría, son el detonante para su desarrollo en la vida adulta (5).

La IR es una respuesta biológica disminuida a la acción de la insulina, lo que lleva a una hiperinsulinemia compensatoria que puede evolucionar, en la mayoría de los casos, a diabetes $(6,7)$. Los reportes de prevalencia en población pediátrica con obesidad son muy amplios, EE.UU. reporta valores de 29.1\%; mientras que en México fue 90\%; en Bolivia 39.4\%; Perú con un $70 \%$ (8-10). Su aparición marca el riesgo de presentar enfermedades endocrino-metabólicas e incluso cardiovasculares con los años. Además, es un predictor de alta 
confiabilidad para el SM, cuya detección precoz podrá evitar la progresión de esta enfermedad y la morbilidad asociada $(5,11)$.

Para evaluar la IR se han utilizado diferentes métodos de análisis. El Clamp Euglicémico Hiperinsulinémico es el estándar de oro para el diagnóstico de esta alteración; aunque, es costoso, muy invasivo, complejo y poco práctico, generalmente es usado con fines de investigación $(4,12)$. El test de tolerancia glucosídica, la poscarga de glucosa y la insulinemia, son usados como métodos alternativos, pero tienen menos confiabilidad debido a los cambios fisiológicos en la población infantil $(2,13)$.

El HOMA-IR es un indicador matemático que ha demostrado tener gran utilidad para la detección y seguimiento de la resistencia insulínica. Actualmente algunos autores han validado esta prueba para identificar la incidencia de SM y DM2 en poblaciones jóvenes, incluso después de varios años $(4,14)$. Otros trabajos demuestran que presenta una moderada a buena correlación con el Clamp (4). Su uso en la práctica clínica se ve limitado por falta de umbrales precisos (ausencia de evidencia longitudinal en pediatría) (14). Muchas investigaciones han marcado sus propios puntos de corte en diferentes poblaciones con el fin de buscar estandarizaciones, evidenciando los estados fisiológicos y patológicos que podrían alterar los resultados (15-17).

El índice evalúa la IR según la relación entre insulinemia y glicemia en ayunas (18); es un método simple, de menor costo, buena sensibilidad en niños y adolescentes. Para establecer su punto de corte es necesario tener en cuenta las variables a las que está sujeto su cálculo $(7,19)$. Este modelo ha sido ampliamente usado en la clínica; además en estudios epidemiológicos y de investigación con muestras grandes $(2,20)$. Su utilidad se ve reflejada en la detección temprana de IR en pacientes obesos y el beneficio para aplicar terapias preventivas para evitar futuras complicaciones (4).

En Ecuador, en el área de pediatría los estudios sobre IR en niños y adolescentes son escasos, a pesar de que los niveles de obesidad, dislipidemias y diabetes infantil son preocupantemente altos. La prevalencia de IR es del $30.3 \%$ en adolescentes y va en aumento. Pocas investigaciones se han llevado a cabo con el uso del HOMA-IR como predictor de IR en población infantil. Por lo tanto, no se ha obtenido un valor de referencia útil para el uso de este parámetro en la clínica y para investigación $(21,22)$. Sin embargo, a pesar que se concluye que el HOMA-IR tiene mayor sensibilidad que la insulina plasmática por sí sola y es más sencillo que otros procedimientos diagnósticos, los resultados obtenidos no se pueden corroborar, pues los registros son insuficientes a nivel nacional (23-24).

Por esta razón la pregunta de investigación es: ¿Cuál es el valor clínico del índice HOMA-IR, como indicador de riesgo, para el desarrollo de enfermedades endocrino-metabólicas frente a otros métodos diagnósticos en la población pediátrica obesa?

Esta revisión tiene como objetivo evaluar el índice HOMA-IR, como indicador de riesgo para el desarrollo de enfermedades endocrino-metabólicas en la población pediátrica con obesidad. Además, mostrar sus limitaciones y utilidad diagnóstica sobre otros métodos para la resistencia a la insulina. 


\section{MÉTODO}

S e realizó una revisión sistemática documental, para lo cual se recopilaron en las bases digitales: Science Direct, Scopus, Scielo y Pubmed, los artículos originales y meta-análisis, que informaron sobre el uso del índice HOMAIR para el diagnóstico de IR en niños y adolescentes obesos. También se analizó su relación con el desarrollo de enfermedades endocrino-metabólicas y sus complicaciones en la edad adulta. La estrategia de búsqueda se restringió para artículos completos en idioma español, inglés y portugués, comprendido entre enero de 2007 y diciembre de 2020.

Esta revisión sistemática se planificó, realizó y se informó de conformidad con los estándares de calidad para revisiones sistemáticas. Para la inclusión y exclusión, los estudios se evaluaron con el método PRISMA. Se presentó en un flujograma la identificación, cribado, elegibilidad, y selección de cada uno (25). Para formular la pregunta de investigación se utilizó la estrategia PICO.

\section{Estrategia de búsqueda}

Los términos de búsqueda que se usaron en la redacción fueron verificados con los descriptores MESH y DeCS, y fueron: "índice HOMA-IR" y "obesidad infantil", "resistencia a la insulina" y "obesidad infantil" y "obesidad infantil" y "riesgos cardiovasculares", en inglés fueron "HOMA-IR" and "Pediatric Obesity", "Insulin resistance" and "Pediatric Obesity" y "Pediatric Obesity" and "Cardiovascular Diseases".

\section{Criterios de inclusión y exclusión}

Se incluyeron los trabajos con los criterios siguientes: ensayos controlados aleatorios, en humanos, edad máxima 18 años, tener reporte de resultados del índice HOMA-IR e individuos de estudio con obesidad. Se evaluaron los marcadores de "resultado de interés": IMC, obesidad, resistencia a la insulina, SM e índice HOMA-IR. Se tomaron en consideración los estudios de puntos de corte del índice HOMA-IR en niños y adolescentes sanos a nivel mundial. Además, se incluyeron aquellos estudios con reporte de sensibilidad y especificidad de la prueba. Se excluyeron los artículos realizados con niños diagnosticados con afecciones endocrinas y cardiovasculares congénitas, y estudios en cualquier otro idioma que no sea español, portugués e inglés.

\section{Extracción y análisis de datos}

Los artículos seleccionados se identificaron mediante el análisis de los títulos, año, resúmenes, discusión y conclusiones. El esquema del proceso y el flujo de trabajo se presentan en la Figura 1. Los estudios elegibles se evaluaron de acuerdo con: el número de participantes, el diseño del estudio, edad, el grado de obesidad, el cálculo del índice HOMA-IR y la presencia y/o ausencia de enfermedades congénitas.

Las concentraciones de biomarcadores registrados se convirtieron a mmol / $\mathrm{L}$ (índice HOMA-IR, glucosa) y $\mu \mathrm{U} / \mathrm{ml}$ (insulina) para estandarizar los resultados según la fórmula de Matthews et al (26). Se realizó una revisión sistemática para combinar los resultados de estudios individuale 


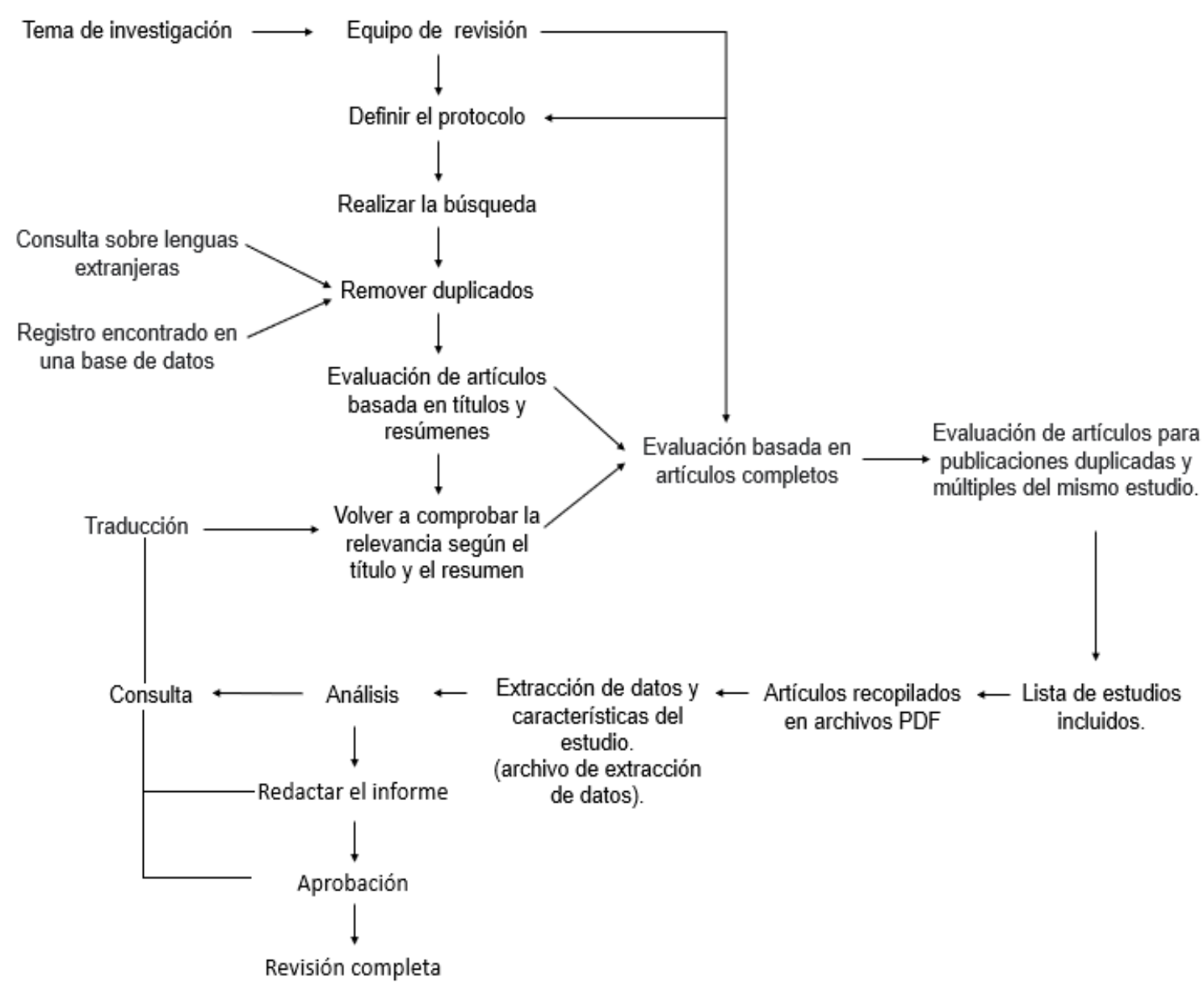

Figura 1.- Diagrama de flujo de proceso. Fuente: Hutton B, et al., (25). La extensión de la declaración PRISMA para revisiones sistemáticas que incorporan metaanálisis en red: PRISMANMA. 2016.

Para evaluar el índice HOMA-IR en el desarrollo de enfermedades endocrinometabólicas en la población pediátrica con obesidad, mostrar sus limitaciones y utilidad diagnóstica, se usaron términos generales y amplios de búsqueda (obesidad pediátrica e índice HOMA-IR). Se localizaron aproximadamente 3855 artículos que fueron evaluados. $\mathrm{Al}$ revisar los títulos y resúmenes, seleccionamos 139 artículos, se eliminaron los duplicados, obteniendo 115 para el análisis. Después de aplicar los criterios de inclusión y exclusión, se asignaron 23 artículos para revisión detallada del texto completo, seleccionando 10 artículos para la extracción y análisis de datos. Ver Figura 2. 


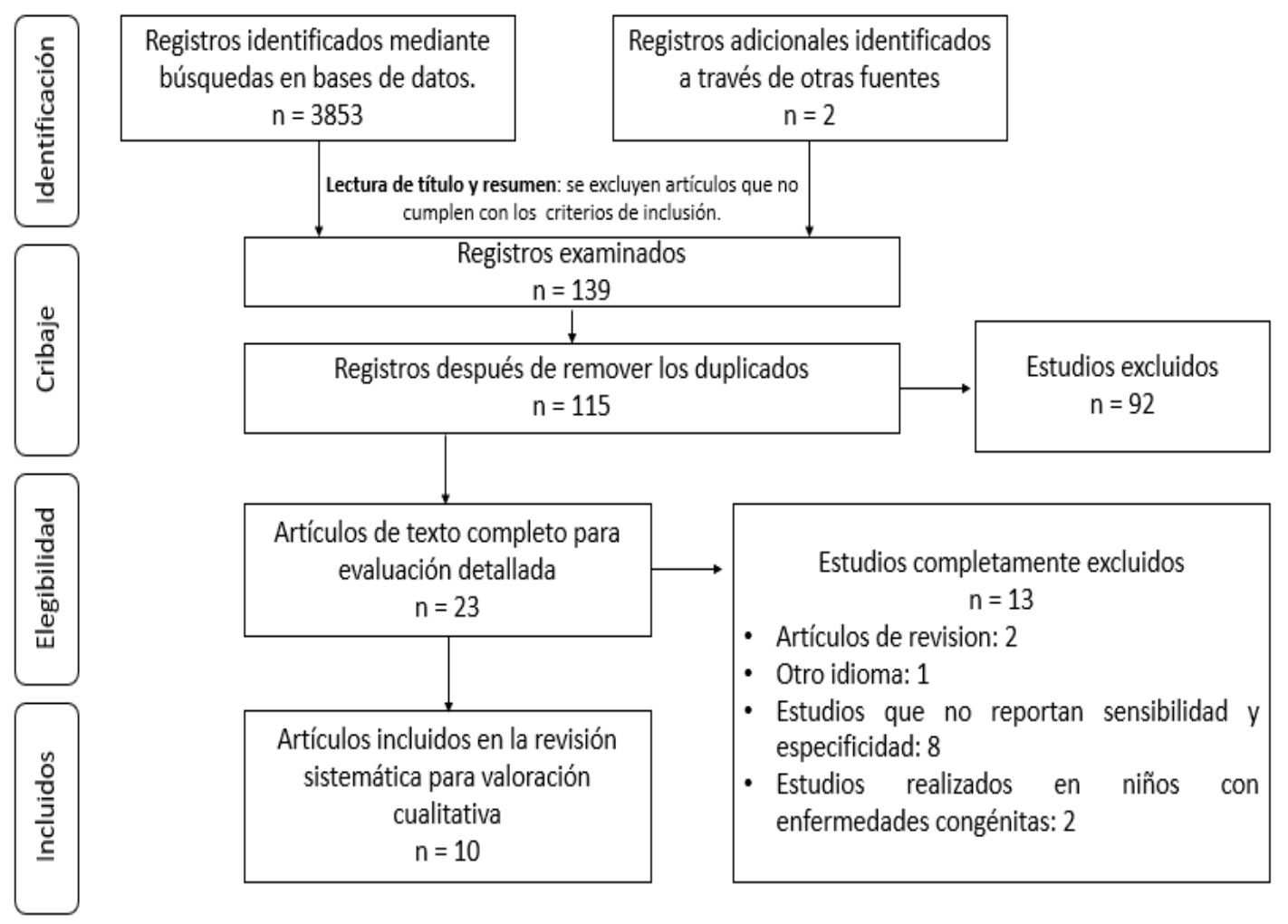

Figura 2.- Diagrama de flujo de la revisión sistemática de la literatura según PRISMA.

de 16,85 puntos, obteniendo un porcentaje de puntuación de calidad superior al $50 \%$.

\section{RESULTADOS}

Las características principales de los artículos incluidos en esta revisión se muestran en la Tabla 1. Se detallan las principales variables bibliométricas de los artículos y se presentan en orden descendente de acuerdo a la puntuación obtenida según los criterios de Fortalecimiento del Reporte de Estudios Observacionales en Epidemiología STROBE (Strengthening the Reporting of Observational studies in Epidemiology) La puntuación de calidad tiene un promedio
Cinco de los estudios (4,27-30) se llevaron a cabo en los continentes de Asia y Europa entre el 2007 y 2016, cuatro (3134 ) en Sudamérica entre los años 2008 y 2020 y uno en Norteamérica (35) en el 2014. La muestra más pequeña fue de 140 niños y adolescentes (31), mientras que la mayor fue de 3203 pacientes (4), con edades comprendidas desde 2 a 18 años. Los estudios incluidos tenían un diseño de cohorte transversal, cuatro de ellos fueron de cohorte anidados $(4,30,32,35)$. 
Tabla 1.- Características principales de los estudios y puntuación de calidad del artículo de acuerdo con los criterios Strobe.

\begin{tabular}{|c|c|c|c|c|c|c|}
\hline TITULO & $\begin{array}{c}\text { AUTOR Y } \\
\text { AÑO } \\
\end{array}$ & FUENTE Y LUGAR & TE & TM & EDAD & PS \\
\hline $\begin{array}{l}\text { Insulin resistance } \\
\text { determined by } \\
\text { Homeostasis } \\
\text { Model Assessment } \\
\text { (HOMA) and } \\
\text { associations with } \\
\text { metabolic } \\
\text { syndrome among } \\
\text { Chinese children } \\
\text { and teenagers }\end{array}$ & $\begin{array}{l}\text { Yin J et al., } \\
(4) \\
2013\end{array}$ & $\begin{array}{l}\text { Beijing - China } \\
\text { Web of Science } \\
\text { Diabetology and } \\
\text { Metabolic } \\
\text { Syndrome } \\
\text { Cuartil: Q2 }\end{array}$ & $\begin{array}{l}\text { Estudio } \\
\text { transversal } \\
\text { cohorte } \\
\text { anidada }\end{array}$ & $\begin{array}{l}\text { Total: } 3203 \\
\text { (1679 varones y } \\
1524 \text { mujeres) }\end{array}$ & 6 a 18 & $\begin{array}{l}19,0 \\
(86,3 \%)\end{array}$ \\
\hline $\begin{array}{l}\text { Cut-off point for } \\
\text { Homeostatic } \\
\text { Model Assessment } \\
\text { for Insulin } \\
\text { Resistance } \\
\text { (HOMA-IR) index } \\
\text { established from } \\
\text { Receiver } \\
\text { Operating } \\
\text { Characteristic } \\
\text { (ROC) curve in the } \\
\text { detection of } \\
\text { metabolic } \\
\text { syndrome in } \\
\text { overweight pre- } \\
\text { pubertal children }\end{array}$ & $\begin{array}{l}\text { Madeira et } \\
\text { al. } \\
(31) \\
2008\end{array}$ & $\begin{array}{l}\text { Rio de Janeiro - } \\
\text { Brasil } \\
\text { Scopus } \\
\text { Arquivos } \\
\text { Brasileiros de } \\
\text { Endocrinologia \& } \\
\text { Metabologia }\end{array}$ & $\begin{array}{l}\text { Estudio } \\
\text { transversal }\end{array}$ & $\begin{array}{l}\text { Total: } 140 \text { niños } \\
\text { y adolescentes } \\
\text { Obesos: } 106 \\
\text { ( } 37 \text { niñas y } 69 \\
\text { niños) } \\
\text { Sobrepeso: } 34 \\
\text { (19 niñas y } 15 \\
\text { niños). }\end{array}$ & 2 a 11 & $\begin{array}{l}18,5 \\
(84,0 \%)\end{array}$ \\
\hline $\begin{array}{l}\text { Reference ranges } \\
\text { of HOMA-IR in } \\
\text { normal-weight } \\
\text { and obese young } \\
\text { Caucasians }\end{array}$ & $\begin{array}{l}\text { Shashaj B et } \\
\text { al. } \\
(27) \\
2016\end{array}$ & $\begin{array}{l}\text { Roma - Italia } \\
\text { Scopus } \\
\text { Acta Diabetológica } \\
\text { Cuartil: Q1 }\end{array}$ & $\begin{array}{l}\text { Estudio } \\
\text { transversal }\end{array}$ & $\begin{array}{l}\text { Total: } 2573 \text { niños } \\
\text { y adolescentes. } \\
\text { (1369 hombres y } \\
1204 \text { mujeres) }\end{array}$ & 2 a 17 & $\begin{array}{l}17,8 \\
(80,9 \%)\end{array}$ \\
\hline $\begin{array}{l}\text { Healthy Chilean } \\
\text { Adolescents with } \\
\text { HOMA-IR } \geq 2.6 \\
\text { Have Increased } \\
\text { Cardiometabolic } \\
\text { Risk: Association } \\
\text { with Genetic, } \\
\text { Biological, and } \\
\text { Environmental } \\
\text { Factors }\end{array}$ & $\begin{array}{l}\text { Burrows R et } \\
\text { al. } \\
(32) \\
2015\end{array}$ & $\begin{array}{l}\text { Santiago - Chile } \\
\text { PubMed } \\
\text { Journal of Diabetes } \\
\text { Research } \\
\text { Cuartil: Q2 }\end{array}$ & $\begin{array}{l}\text { Estudio } \\
\text { transversal } \\
\text { cohorte } \\
\text { anidada }\end{array}$ & $\begin{array}{l}\text { Total: } 667 \\
(52,2 \% \text { de } \\
\text { varones y un } \\
47,8 \% \text { de } \\
\text { mujeres) }\end{array}$ & 16 a 17 & $\begin{array}{l}17,3 \\
(78,6 \%)\end{array}$ \\
\hline $\begin{array}{l}\text { High HOMA-IR, } \\
\text { adjusted for } \\
\text { puberty, relates to } \\
\text { the metabolic } \\
\text { syndrome in } \\
\text { overweight and } \\
\text { obese Chilean } \\
\text { youths }\end{array}$ & $\begin{array}{l}\text { Burrows R et } \\
\text { al. } \\
(33) \\
2011\end{array}$ & $\begin{array}{l}\text { Santiago - Chile } \\
\text { PubMed } \\
\text { Pediatric Diabetes } \\
\text { Cuartil: Q1 }\end{array}$ & $\begin{array}{l}\text { Estudio } \\
\text { transversal }\end{array}$ & $\begin{array}{l}\text { Total: } 486 \\
\text { jóvenes con } \\
\text { sobrepeso y } \\
\text { obesidad }\end{array}$ & 5 a 15 & $\begin{array}{l}17,0 \\
(77,2 \%)\end{array}$ \\
\hline
\end{tabular}




\begin{tabular}{|c|c|c|c|c|c|c|}
\hline TITULO & $\begin{array}{c}\text { AUTOR Y } \\
\text { AÑO }\end{array}$ & FUENTE Y LUGAR & TE & TM & EDAD & PS \\
\hline $\begin{array}{l}\text { Screening Obese } \\
\text { Children and } \\
\text { Adolescents for } \\
\text { Prediabetes } \\
\text { and/or Type } 2 \\
\text { Diabetes in } \\
\text { Pediatric } \\
\text { Practices: A } \\
\text { Validation Study }\end{array}$ & $\begin{array}{l}\text { Brar P et al. } \\
(35) \\
2014\end{array}$ & $\begin{array}{l}\text { New York- } \\
\text { Estados Unidos } \\
\text { PubMed } \\
\text { Clinical Pediatrics } \\
\text { Cuartil: Q2 }\end{array}$ & $\begin{array}{l}\text { Estudio } \\
\text { transversal } \\
\text { cohorte } \\
\text { anidada }\end{array}$ & $\begin{array}{l}\text { Total: } 149 \text { niños } \\
\text { y adolescentes } \\
\text { obesos } \\
\text { (relativamente } \\
\text { sanos } 125, \\
\text { prediabéticos } 21 \\
\text { y diabéticos } 3 \text { ) }\end{array}$ & 10 a 16 & $\begin{array}{l}16,8 \\
(76,3 \%)\end{array}$ \\
\hline $\begin{array}{l}\text { Insulin Resistance } \\
\text { in Obese Children } \\
\text { and Adolescents: } \\
\text { HOMA-IR Cut-Off } \\
\text { Levels in the } \\
\text { Prepubertal and } \\
\text { Pubertal Periods }\end{array}$ & $\begin{array}{l}\text { Kurtoğlu S et } \\
\text { al. } \\
(28) \\
2010\end{array}$ & $\begin{array}{l}\text { Kayseri -Turquía } \\
\text { PubMed } \\
\text { Journal of Clinical } \\
\text { Research in } \\
\text { Pediatric } \\
\text { Endocrinology } \\
\text { Cuartil: Q2 }\end{array}$ & $\begin{array}{l}\text { Estudio } \\
\text { transversal }\end{array}$ & $\begin{array}{l}\text { Total: } 268 \text { niños } \\
\text { y adolescentes } \\
\text { (141 niñas con } \\
\text { obesidad, } 127 \\
\text { niños con } \\
\text { obesidad) }\end{array}$ & 5 a 18 & $\begin{array}{l}16,5 \\
(75,0 \%)\end{array}$ \\
\hline $\begin{array}{l}\text { Assessment of } \\
\text { Insulin Sensitivity } \\
\text { from } \\
\text { Measurements in } \\
\text { Fasting State and } \\
\text { During an Oral } \\
\text { Glucose Tolerance } \\
\text { Test in Obese } \\
\text { Children }\end{array}$ & $\begin{array}{l}\text { Atabek M et } \\
\text { al. } \\
(29) \\
2007\end{array}$ & $\begin{array}{l}\text { Konya - Turquía } \\
\text { Scopus } \\
\text { Journal of Pediatric } \\
\text { Endocrinology and } \\
\text { Metabolism } \\
\text { Cuartil: Q2 }\end{array}$ & $\begin{array}{l}\text { Estudio } \\
\text { transversal }\end{array}$ & $\begin{array}{l}\text { Total: } 148 \text { niños } \\
\text { y adolescentes } \\
\text { Sin IR: } 93 \text { niños y } \\
\text { adolescentes } \\
\text { obesos ( } 54 \text { niñas } \\
\text { y } 39 \text { niños) } \\
\text { Con IR: } 55 \text { niños } \\
\text { y adolescentes } \\
\text { obesos ( } 32 \text { niñas } \\
\text { y } 23 \text { niños) }\end{array}$ & 7 a 13 & $\begin{array}{l}15,8 \\
(71,8 \%)\end{array}$ \\
\hline $\begin{array}{l}\text { Adiponectin, } \\
\text { HOMA- } \\
\text { Adiponectin, } \\
\text { HOMA-IR in } \\
\text { Children and } \\
\text { Adolescents: Ouro } \\
\text { Preto Study }\end{array}$ & $\begin{array}{l}\text { Cândido A et } \\
\text { al. } \\
(34) \\
2020\end{array}$ & $\begin{array}{l}\text { Minas Gerais - } \\
\text { Brazil Scopus } \\
\text { Indian Journal of } \\
\text { Pediatrics } \\
\text { Cuartil: Q3 }\end{array}$ & $\begin{array}{l}\text { Estudio } \\
\text { transversal }\end{array}$ & $\begin{array}{l}\text { Total 691: } 257 \\
\text { niños y } 434 \\
\text { adolescentes. } \\
\text { (354 mujeres y } \\
\text { 337 hombres) }\end{array}$ & 6 a 14 & $\begin{array}{l}15,5 \\
(70,4 \%)\end{array}$ \\
\hline $\begin{array}{l}\text { A Study of Insulin } \\
\text { Resistance by } \\
\text { HOMA-IR and its } \\
\text { Cut-off Value to } \\
\text { Identify Metabolic } \\
\text { Syndrome in } \\
\text { Urban Indian } \\
\text { Adolescents }\end{array}$ & $\begin{array}{l}\text { Singh Y et al. } \\
(30) \\
2013\end{array}$ & $\begin{array}{l}\text { Delhi - India } \\
\text { PubMed } \\
\text { Journal of Clinical } \\
\text { Research in } \\
\text { Pediatric } \\
\text { Endocrinology } \\
\text { Cuartil: Q2 }\end{array}$ & $\begin{array}{l}\text { Estudio } \\
\text { transversal } \\
\text { cohorte } \\
\text { anidada }\end{array}$ & $\begin{array}{l}\text { Total: } 691 \\
\text { adolescentes. } \\
\text { (199 obesos, } 205 \\
\text { con sobrepeso y } \\
295 \text { adolescentes } \\
\text { de peso normal). }\end{array}$ & 10 a 17 & $\begin{array}{l}14,3 \\
(65,0 \%)\end{array}$ \\
\hline
\end{tabular}

TE: Tipo de Estudio; TM: Tamaño de la Muestra; PS: Puntuación STROBE; HOMA-IR: modelo de homeostasis de resistencia a la insulina; EM: enfermedad metabólica; IR: resistencia a la insulina; CMRF: factores de riesgo cardiometabólicos; SM: síndrome metabólico.

Los participantes incluidos en los estudios seleccionados presentaban un grado de sobrepeso u obesidad según la evaluación de la puntuación Z del IMC. Los puntos de corte del HOMA-IR varían dependiendo de la edad, estado nutricional, sexo y grado de maduración sexual, el más bajo fue de 1,7 y el más alto 5,2 Gráfico 1. 


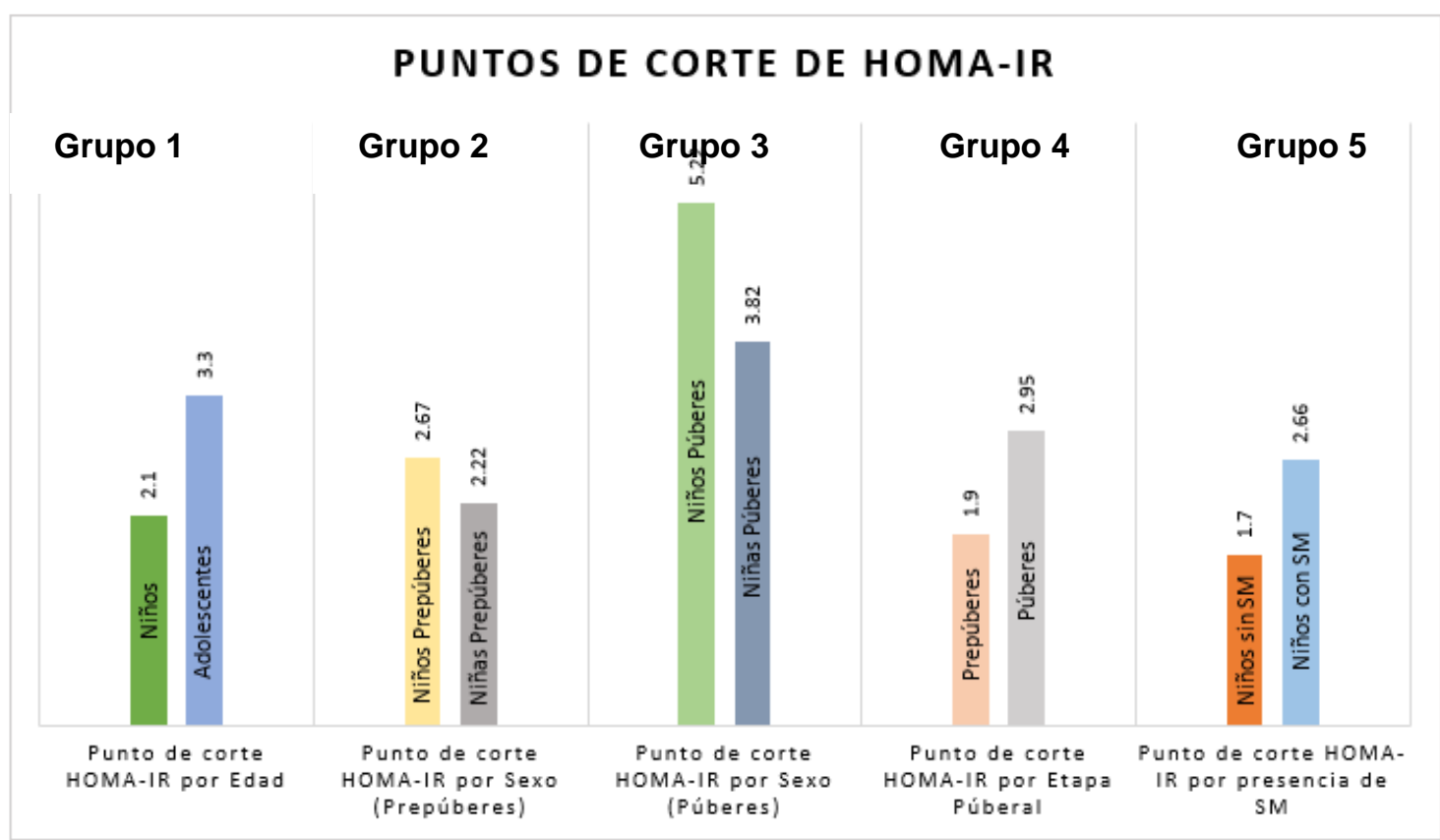

Gráfico 1.-Punto de corte del índice HOMA-IR según diferentes artículos incluidos en la revisión. Las barras del grupo 1 corresponden a la comparación del HOMA-IR entre niños y adolescentes propuesto por Burrows R et al., (33). En el grupo 2 y 3 se compara el HOMA-IR entre niños y niñas tanto púberes como prepúberes planteado por Kurtoğlu S et al, (28). El grupo 4 es una comparación por etapa puberal propuesta por Yin J et al., (4). El grupo 5 se compara pacientes pediátricos saludables con pacientes con uno o más componentes de SM hallados por Yin J et al., y Burrows R et al., $(4,32)$.

De los diez artículos incluidos, dos de ellos $(4,28)$ proporcionaron información sobre las etapas de maduración sexual (púberes y prepúberes) para el punto de corte. Dos estudios hacen una diferenciación entre el corte para niños y adolescentes $(33,34)$. Uno de ellos informa sobre la diferencia de género y etapa de maduración sexual (28). Los demás artículos $(27,29,31,32,34,35)$ muestran el punto de corte óptimo para el diagnóstico de IR, SM, CMRF, prediabetes y diabetes, ver Tabla 2. 
Tabla 2.- Puntos de corte, sensibilidad y especificidad del índice HOMA-IR según el tipo de población analizada.

\begin{tabular}{|c|c|c|c|c|}
\hline ARTÍ́CULO & IMC & $\begin{array}{l}\text { PC DEL ÍNDICE } \\
\text { HOMA-IR }\end{array}$ & $\begin{array}{l}\text { SENSIBILIDAD } \\
\text { DEL MÉTODO }\end{array}$ & $\begin{array}{l}\text { ESPECIFICIDAD } \\
\text { DEL MÉTODO }\end{array}$ \\
\hline $\begin{array}{l}\text { Insulin resistance } \\
\text { determined by } \\
\text { Homeostasis Model } \\
\text { Assessment (HOMA) } \\
\text { and associations with } \\
\text { metabolic syndrome } \\
\text { among Chinese children } \\
\text { and teenagers }\end{array}$ & $\begin{array}{l}\text { En población normal: } \\
18,72 \pm 3,36 \mathrm{~kg} / \mathrm{m}^{2} \text {. } \\
\text { Más de } 3 \\
\text { componentes de EM: } \\
27,66 \pm 4,11 \mathrm{~kg} / \mathrm{m}^{2} \text {. }\end{array}$ & $\begin{array}{l}\text { Pacientes con EM: } \\
\text { Todos: } 2,3 \\
\text { Prepúberes: } 1,7 \\
\text { Púberes; } 2,6 \text {. } \\
\text { Pacientes normales: } \\
\text { Todos: } 3,0 \\
\text { Prepúberes: } 2,6 \\
\text { Púberes: } 3,2 \text {. }\end{array}$ & $\begin{array}{l}\text { Pacientes con EM: } \\
\text { Todos: } 80 \% \\
\text { Prepúberes: } 86 \% \\
\text { Púberes: } 78 \% \\
\text { Pacientes } \\
\text { normales: Todos: } \\
\text { 57\% Prepúberes: } \\
\text { 54\% Púberes: } 67 \%\end{array}$ & $\begin{array}{l}\text { Pacientes con EM: } \\
\text { Todos: } 66 \% \\
\text { Prepúberes: } 67 \% \\
\text { Púberes: } 67 \% \\
\text { Pacientes } \\
\text { normales: Todos: } \\
\text { 83\% Prepúberes: } \\
\text { 85\% Púberes: } 80 \%\end{array}$ \\
\hline $\begin{array}{l}\text { Cut-off point for } \\
\text { Homeostatic Model } \\
\text { Assessment for Insulin } \\
\text { Resistance (HOMA-IR) } \\
\text { index established from } \\
\text { Receiver Operating } \\
\text { Characteristic (ROC) } \\
\text { curve in the detection of } \\
\text { metabolic syndrome in } \\
\text { overweight pre-pubertal } \\
\text { children }\end{array}$ & $\begin{array}{l}\text { Puntuación } \mathrm{Z} \text { de IMC: } \\
2,1 \pm 0,7\end{array}$ & $\begin{array}{l}\text { Punto de corte } \\
\text { óptimo para SM: } 2,5\end{array}$ & $\begin{array}{l}\text { Sensibilidad: } \\
60,8 \%\end{array}$ & $\begin{array}{l}\text { Especificidad: } \\
83,7 \%\end{array}$ \\
\hline $\begin{array}{l}\text { Reference ranges of } \\
\text { HOMA-IR in normal- } \\
\text { weight and obese young } \\
\text { Caucasians }\end{array}$ & $\begin{array}{l}808 \text { participantes } \\
\text { IMC B percentil } 85 \text { y } \\
1765 \text { tenían } \\
\text { sobrepeso / obesidad }\end{array}$ & $\begin{array}{l}\text { Punto de corte } \\
\text { óptimo para CMRF: } \\
3,02\end{array}$ & $\begin{array}{l}\text { Sensibilidad: } \\
46.3 \%\end{array}$ & $\begin{array}{l}\text { Especificidad: } \\
82,6 \%\end{array}$ \\
\hline $\begin{array}{l}\text { Healthy Chilean } \\
\text { Adolescents with } \\
\text { HOMA-IR } \geq 2.6 \text { Have } \\
\text { Increased } \\
\text { Cardiometabolic Risk: } \\
\text { Association with } \\
\text { Genetic, Biological, and } \\
\text { Environmental Factors }\end{array}$ & $\begin{array}{l}\text { IMC (puntuación z): } \\
\text { Pacientes HOMA-IR: } \\
\geq 1,53 \pm 1,2 \text { Muestra } \\
\text { total: } 0,65 \pm 1,2\end{array}$ & $\begin{array}{l}\text { Punto de corte } \\
\text { óptimo para MetS: } \\
2,6\end{array}$ & Sensibilidad: $59 \%$ & Especificidad: $87 \%$ \\
\hline $\begin{array}{l}\text { High HOMA-IR, adjusted } \\
\text { for puberty, relates to } \\
\text { the metabolic syndrome } \\
\text { in overweight and obese } \\
\text { Chilean youths }\end{array}$ & $\begin{array}{l}\text { IMC (puntuación z): } \\
\text { Etapa I -II Tanner: } \\
\text { hombres: } 4,1 \text {. } \\
\text { mujeres: } 3,6 \\
\text { Etapa III -V Tanner: } \\
\text { hombres: } 2,8 \\
\text { mujeres: } 2,9\end{array}$ & $\begin{array}{l}\text { Punto de corte } \\
\text { óptimo para MetS: } \\
\text { Etapa I -II Tanner } \\
\text { niños: } 2,1 \text {. } \\
\text { Etapa III -V Tanner } \\
\text { adolescentes: } 3,3\end{array}$ & Sensibilidad: $65 \%$ & Especificidad: $69 \%$ \\
\hline
\end{tabular}




\begin{tabular}{|c|c|c|c|c|}
\hline ARTÍCULO & IMC & $\begin{array}{l}\text { PC DEL ÍNDICE } \\
\text { HOMA-IR }\end{array}$ & $\begin{array}{l}\text { SENSIBILIDAD } \\
\text { DEL MÉTODO }\end{array}$ & $\begin{array}{l}\text { ESPECIFICIDAD } \\
\text { DEL MÉTODO }\end{array}$ \\
\hline $\begin{array}{l}\text { Screening Obese } \\
\text { Children and } \\
\text { Adolescents for } \\
\text { Prediabetes and/or } \\
\text { Type } 2 \text { Diabetes in } \\
\text { Pediatric Practices: A } \\
\text { Validation Study }\end{array}$ & $\begin{array}{l}\text { IMC (puntuación z): } \\
\text { Normal: } 2.3 \pm 0.5 \\
\text { Prediabetes: } 2.1 \pm 0.7 \\
\text { Diabetes: } 2.1 \pm 0.5\end{array}$ & $\begin{array}{l}\text { Punto de corte para } \\
\text { prediabetes / DM: } 3,4\end{array}$ & Sensibilidad: $65 \%$ & $\begin{array}{l}\text { Especificidad: } \\
60,7 \%\end{array}$ \\
\hline Insulin Resistance in & Prepúberes: & Prepúberes: & Prepúber: & Prepúber: \\
\hline Obese Children and & niños: $28,2 \pm 5,4$ & Niños: 2,67 & Niños: $88,2 \%$ & Niños: $65,5 \%$ \\
\hline Adolescents: HOMA-IR & $\mathrm{kg} / \mathrm{m} 2$ & Niñas: 2,22 & Niñas: $100 \%$ & Niñas: $42,3 \%$ \\
\hline Cut-Off Levels in the & niñas: $26,2 \pm 5,8$ & Púberes: & Púberes: & \\
\hline Prepubertal and & $\mathrm{kg} / \mathrm{m} 2$ & Niños: 5,22 & Niños: 56\% & Púberes: \\
\hline Pubertal Periods & $\begin{array}{l}\text { Púberes: } \\
\text { niños: } 30,9 \pm 4,9 \\
\mathrm{~kg} / \mathrm{m} 2 \\
\text { niñas: } 30,4 \pm 5,0 \\
\mathrm{~kg} / \mathrm{m} 2\end{array}$ & Niñas: 3,82 & Niñas: $77,1 \%$ & $\begin{array}{l}\text { Niños: } 93,3 \% \\
\text { Niñas: } 71,4 \%\end{array}$ \\
\hline $\begin{array}{l}\text { Assessment of Insulin } \\
\text { Sensitivity from } \\
\text { Measurements in } \\
\text { Fasting State and During } \\
\text { an Oral Glucose } \\
\text { Tolerance Test in Obese } \\
\text { Children }\end{array}$ & $\begin{array}{l}\text { Con IR: } 27 \pm 4.2 \\
\mathrm{~kg} / \mathrm{m} 2 \\
\text { Sin IR: } 27.9 \pm 3.9 \\
\mathrm{~kg} / \mathrm{m} 2\end{array}$ & $\begin{array}{l}\text { Punto de corte para } \\
\text { IR: }>2,7\end{array}$ & Sensibilidad: $80 \%$ & $\begin{array}{l}\text { Especificidad: } \\
59,1 \%\end{array}$ \\
\hline $\begin{array}{l}\text { Adiponectin, HOMA- } \\
\text { Adiponectin, HOMA-IR } \\
\text { in Children and } \\
\text { Adolescents: Ouro Preto } \\
\text { Study }\end{array}$ & $\begin{array}{l}\text { IMC (puntuación } \mathrm{z} \text { ): } \\
\text { Normal: } 0.10 \pm 1.1 \\
\text { Niños: } 0.21 \pm 1.1 \\
\text { Adolescentes: } 0.04 \pm \\
1.1\end{array}$ & $\begin{array}{l}\text { Niños: }>2.4 \\
\text { Adolescentes: }>2.2\end{array}$ & $\begin{array}{l}\text { Niños: } \\
\text { Sensibilidad: 80\% } \\
\text { Adolescentes: } \\
\text { Sensibilidad: 80\% }\end{array}$ & $\begin{array}{l}\text { Niños: } \\
\text { Especificidad: } \\
96,7 \% \\
\text { Adolescentes: } \\
\text { Especificidad: } 80 \%\end{array}$ \\
\hline $\begin{array}{l}\text { A Study of Insulin } \\
\text { Resistance by HOMA-IR } \\
\text { and its Cut-off Value to } \\
\text { Identify Metabolic } \\
\text { Syndrome in Urban } \\
\text { Indian Adolescents }\end{array}$ & $\begin{array}{l}\text { IMC (puntuación z): } \\
\text { Niños: 22,8 } \pm 5,6 \\
\mathrm{~kg} / \mathrm{m} 2 \\
\text { Niñas: } 23,8 \pm 5,8 \\
\mathrm{~kg} / \mathrm{m} 2\end{array}$ & $\begin{array}{l}\text { Punto de corte para } \\
\text { IR: }>2,5\end{array}$ & $\begin{array}{l}\text { Sensibilidad: } \\
>70 \% \text { para } \mathrm{SM} .\end{array}$ & $\begin{array}{l}\text { Especificidad: } \\
>60 \% \text { para } S M\end{array}$ \\
\hline
\end{tabular}

PC: Punto de Corte; HOMA-IR: Modelo de Homeostasis de Resistencia a la Insulina; EM: Enfermedad Metabólica; IR: Resistencia a la Insulina; CMRF: Factores de Riesgo Cardiometabólicos; SM: Síndrome Metabólico.

Para establecer el punto de corte, la sensibilidad y especificidad, los estudios usaron la Curva de Características Operativas del Receptor (ROC) como herramienta. Se halló que la sensibilidad en la mayoría de los estudios fue superior al 65\%, exceptuando tres artículos $(27,31,32)$ en los que se mostró porcentajes menores. La especificidad en los artículos revisados fue superior al $70 \%$ excepto en cuatro investigaciones (29,30,33,35), ver Gráfico 2.
La menor sensibilidad calculada fue de $38,2 \%$ fue encontrada en niños $y$ adolescentes obesos para determinar el riesgo de $\mathrm{CMRF}$, mientras que la más alta fue del $100 \%$ en niñas prepúberes para determinar IR. La especificidad más baja encontrada para el HOMA-IR fue de 65,5\% en niños prepúberes mientras que la más alta fue de $96,7 \%$ en niños obesos para determinar riesgo de SM. 


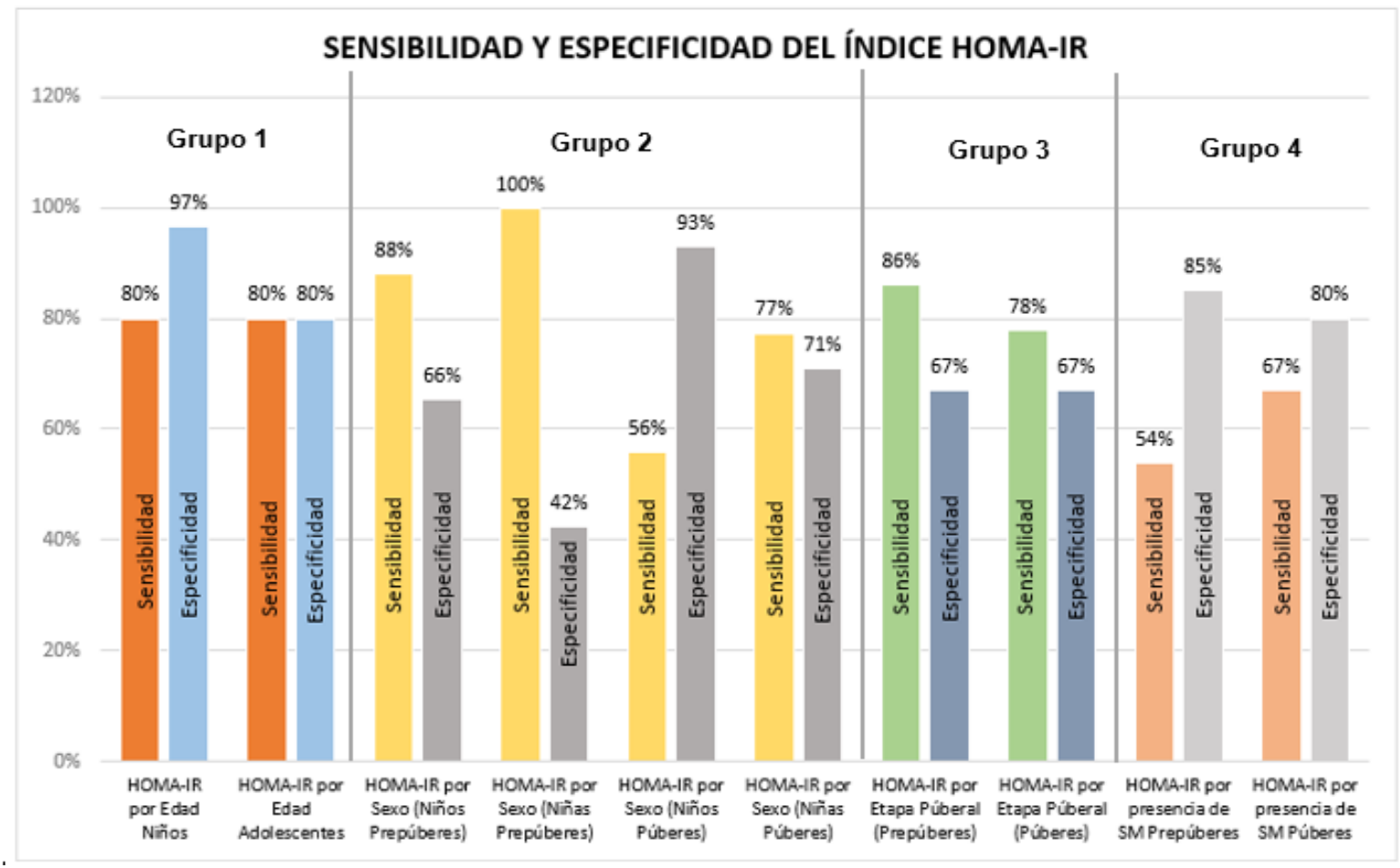

Gráfico 2.-Sensibilidad y Especificidad del índice HOMA-IR según diferentes artículos incluidos en la revisión. Las barras del grupo 1 corresponden a los porcentajes de sensibilidad y especificidad del HOMA-IR entre niños y adolescentes propuesto por Cândido A et al. (34). En el grupo 2 se compara la sensibilidad y especificidad del HOMA-IR entre niños y niñas tanto púberes como prepúberes planteado por Kurtoğlu S et al. (28). El grupo 3 es una comparación entre pacientes púberes y prepúberes con uno o más componentes de SM propuesta por Yin J et al., (4). El grupo 4 compara el porcentaje entre pacientes pediátricos púberes y prepúberes saludables hallado por Yin J et al., (4).

Las limitaciones principales en los artículos incluidos fueron: tamaño pequeño de las muestras, sin cálculo muestral y representatividad, con la imposibilidad de validación externa. Puntos de corte HOMAIR inespecíficos para género, edad, etnia y etapa de maduración sexual. Poca evidencia clínica de enfermedades crónicas en pediatría y la inexistencia de un consenso sobre la definición de SM para niños y adolescentes. La falta de estandarización del método en los laboratorios para determinar IR y comparación con los métodos validados.

\section{DISCUSIÓN}

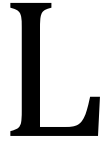
a obesidad es cada vez más frecuente en niños y adolescentes a nivel mundial. Esta alteración está estrechamente relacionada con la aparición de IR, que es una patología multifactorial que aumenta el riesgo de padecer enfermedades crónicas como DM2 y SM. Todas estas afecciones pueden ocasionar complicaciones cardiovasculares en la edad adulta (31,36-37). 
Varios estudios reportan aumento del HOMA-IR en niños y adolescentes con sobrepeso u obesidad en comparación de aquellos con un IMC normal (32), incluso se plantea que puede ser independiente de la edad (27). Investigaciones demuestran que el aumento de la puntuación $\mathrm{Z}$ del IMC junto con un HOMA-IR alto se asocian directamente con el riesgo cardiovascular, ya que la obesidad favorece a la aparición de IR $(29,33)$. Esto enfatiza que el diagnóstico temprano de IR en niños en la etapa puberal es esencial.

El HOMA-IR puede sufrir inconsistencias por factores como edad, sexo y etapa puberal. Se ha reportado que los valores del índice HOMA son diferentes en prepúberes y púberes $(28,31,33)$. En adolescentes se produce una IR transitoria considerada fisiológica, algunos autores plantean que es por un cambio hormonal de esta etapa, especialmente producido por la hormona del crecimiento (34). Sin embargo este cambio no se comprende completamente hasta el momento (28).

Se sabe que el índice HOMA-IR alcanza su nivel máximo en la etapa 3-4 de Tanner y posteriormente disminuye en un $30 \%$ en la etapa 5 (4). Esta situación representa un periodo de mayor riesgo para el desarrollo de DM2, ya que induce a un estrés adicional en las células beta pancreáticas (28-29,31). Los investigadores han marcado diferentes puntos de corte para este modelo, tomando en cuenta etnia, peso, edad, sexo y estadio de Tanner $(4,30$ $31,33,34)$, mostrando resultados muy variados.

$\mathrm{Al}$ analizar la diferencia de género para el índice HOMA, se observa que sus valores suelen ser menores en adolescentes varones (38). Esta situación puede deberse a que en las mujeres, por la menarquia y maduración sexual, hay una redistribución de la grasa corporal, aumentando en el tronco y abdomen, y a su vez produce un aumento significativo del HOMA (21). Kurtoğlu, et al., (28) en su investigación establece una comparación de puntos de corte en niños y niñas en etapas prepuberal y puberal, obteniendo resultados mayores en niños. Otros estudios $(4,28,34)$, corroboran esta información, pues el corte del HOMA-IR varía significativamente en adolescentes mujeres frente a valores menores en varones. Por tanto, es indispensable que en los estudios en adolescentes se tome en cuenta el sexo.

De los estudios recopilados, se concluye que un HOMA-IR alto se relaciona con un aumento de riesgo de SM. Yin, et al., (4) demostró que los niños y adolescentes en el quintil más alto de HOMA-IR tienen 60 veces más probabilidades de presentar este síndrome. El SM se encontró con mayor frecuencia en niños y adolescentes con IR, lo que indica que las anomalías metabólicas asociadas con SM son el resultado a largo plazo de esta patología $(30-32,34)$.

Los estudios realizados por Yin, et al., (4) y Shashaj, et al., (27) en poblaciones amplias, teniendo en cuenta el peso, edad y sexo, proporciono puntos de corte con amplia sensibilidad y especificidad para identificar pacientes pediátricos con alto riesgo de SM. Madeira, et al., (31) indica que los niños y adolescentes con características de SM se convierten en adultos con SM con riesgo de patologías cardiovasculares ateroscleróticas.

Actualmente se sabe que la incidencia de DM2 es cada vez mayor en niños, especialmente en aquellos con sobrepeso y obesidad. Como ya se explicó, la adolescencia, por la IR fisiológica transitoria, es un periodo de alto riesgo 
para el desarrollo de DM (28). Brar, et al. (35), realizó un estudio de comparación del índice HOMA-IR entre pacientes sanos, con prediabetes y diabetes, marcando diferentes puntos de corte. Concluyó que la DM2 no se encontró con mayor incidencia en la población estudiada, sin embargo, enfatizó que el HOMA-IR no es suficientemente preciso como prueba única para la detección de prediabetes (28).

La curva ROC es un método estadístico que se usa para establecer un punto de corte óptimo teniendo en cuenta la sensibilidad y especificidad. Estos parámetros indican que la prueba distribuye correctamente a los pacientes estudiados en enfermos (positivo) y no enfermos (negativo) respectivamente $(39,40)$. Para que una prueba sea válida para la detección de una patología, debe tener una sensibilidad alta y una especificidad moderada, sin embargo, para diagnóstico el test necesita una especificidad mayor (29).

El clamp euglucémico es el estándar de oro para el diagnóstico de IR, pero es un método costoso, invasivo y complejo, casi no se usa en la práctica diaria $(4,41)$. Estudios anteriores han demostrado que el índice HOMA-IR tiene una buena correlación con el clamp para la detección temprana de IR $(4,30,41-42)$ sin embargo, está sujeto a varias limitaciones. La principal es la falta de un punto de corte estandarizado en la población pediátrica y las variables fisiológicas y patológicas que pueden alterar sus valores (25-26).

De los estudios incluidos en esta revisión, todos establecieron su propio punto de corte para la alteración que investigaban. Los autores de ocho de los artículos revisados optaron por el uso de puntos de corte con mejor sensibilidad y especificidad $(4,28-33,35)$, en dos de los artículos se prefirió trabajar con un punto de corte más conveniente para la detección de $\operatorname{SM}(27,34)$.

De la información analizada se puede inferir que el punto de corte con mayor sensibilidad fue el propuesto por Kurtoğlu $S$ et al. (28), para la detección precoz de IR en niñas prepúberes, lo que aumenta la probabilidad de SM y complicaciones cardiovasculares. La mayor especificidad encontrada fue propuesta por Cándido A et al., (34) para descartar la presencia de SM en pacientes pediátricos. Se puede observar amplias discordancias en los valores de sensibilidad y especificidad del HOMA, debido a las características desiguales de las poblaciones estudiadas. Cabe mencionar que la disminución de la curva ROC en varios artículos $(27,32$ 33,35 ) puede deberse al diseño de estudio y no directamente a una utilidad clínica deficiente del índice HOMA-IR.

En el desarrollo de la revisión se presentaron varias limitantes: no todos los estudios tienen muestras representativas, ninguno de ellos permite una comparación de resultados de HOMA-IR frente al clamp euglucémico, no se han establecido un punto de corte propiamente dicho para insulinemia y HOMA-IR en pediatría según edad, sexo y maduración sexual, por lo que no se puede hacer una comparación entre los artículos originales.

Se recomienda que en futuras investigaciones se realice una comparación entre las diferentes pruebas diagnósticas de IR frente al HOMA-IR, especialmente el clamp euglucémico que es el estándar de oro. También se debería evaluar los puntos de corte entre poblaciones pediátricas relativamente sanas $y$ aquellas que presenten comorbilidades, para tener una mayor visión de la utilidad del índice. 
Además se requiere profundizar los estudios de IR en niños y adolescentes según edad, sexo y grado de maduración sexual, ya que estos factores producen importantes cambios en el metabolismo.

\section{CONCLUSIÓN}

$\mathrm{L}$ a obesidad está directamente relacionada con el desarrollo de IR en la infancia. El índice HOMA-IR es un método confiable para el diagnóstico de IR temprana en niños y adolescentes con diferentes grados de obesidad, debido a su alta especificidad. Es un modelo simple, de bajo costo y poco invasivo, que permite una intervención terapéutica preventiva y diagnóstica. Utilizando puntos de corte que se adapten mejor a la población estudiada, se vuelve un excelente predictor de enfermedades endocrino-metabólicas y complicaciones cardiovasculares futuras. Para su uso se debe tener en cuenta el peso, edad, género, etnia y etapa de maduración sexual, ya que estos factores generan variación en sus resultados. Además, su sensibilidad permite identificar de mejor manera a los grupos en riesgo y tomar medidas oportunas, como proyectos de intervención en la edad escolar y propuestas educativas que puedan evitar las complicaciones tanto en la adolescencia como en la adultez.

\section{REFERENCIAS BIBLIOGRÁFICAS}

1. OMS. Obesidad y sobrepeso [Internet]. Organización Mundial de la Salud. 2020 [citado 16 de diciembre de 2020]. Disponible en: https://www.who.int/es/news-room/factsheets/detail/obesity-and-overweight

2. Picos S, Pérez C. Resistencia insulínica y los componentes del síndrome metabólico en niños y adolescentes obesos. Rev Cuba Pediatría [Internet]. [citado 12 de diciembre de 2020]; 2015;87(4):449-59. Disponible en: http://scielo.sld.cu/scielo.php?script=sci_a bstract\&pid=S0034-

$75312015000400007 \& \operatorname{lng}=e s \& n r m=i s o \& t$ lng=es

3. Nogueira C, De Mello E. Different Criteria for the Definition of Insulin Resistance and Its Relation with Dyslipidemia in Overweight and Obese Children and Adolescents. Pediatr Gastroenterol Hepatol Nutr [Internet]. [citado 12 de diciembre de 2020]; 2018;21(1):59-67. Disponible en: https://www.ncbi.nlm.nih.gov/pmc/articl es/PMC5788952/

4. Yin J, Li M, Xu L, Wang Y, Cheng H, Zhao $\mathrm{X}$, et al. Insulin resistance determined by Homeostasis Model Assessment (HOMA) and associations with metabolic syndrome among Chinese children and teenagers. Diabetol Metab Syndr [Internet]. 2013;5:71. Disponible en: https://appswebofknowledge-

com.vpn.ucacue.edu.ec/full_record.do?pro duct $=$ WOS\&search_mode $=$ GeneralSearch \& qid=1\&SID=7AJE1zZjxDYuc9qy9TU\&page $=1 \&$ doc $=1$

5. Del Aguila C. Obesidad en el niño: factores de riesgo y estrategias para su prevención en Perú. Rev Peru Med Exp Salud Pública [Internet]. [citado 12 de diciembre de 2020]; 2017;34(1):113-8. Disponible en: http://www.scielo.org.pe/scielo.php?scrip $\mathrm{t}=$ sci_abstract $\&$ pid=S1726-

$46342017000100016 \& \operatorname{lng}=\mathrm{es} \& \mathrm{nrm}=\mathrm{iso} \& \mathrm{t}$ $\operatorname{lng}=\mathrm{es}$

6. Sapunar J, Aguilar-Farías N, Navarro J, Araneda G, Chandia-Poblete D, Manríquez $\mathrm{V}$, et al. High prevalence of overweight, obesity, insulin resistance and metabolic syndrome in rural children and adolescents. Rev Médica Chile [Internet]. [citado 12 de diciembre de 2020]; 
2018;146(9):978-86. Disponible en: https://scielo.conicyt.cl/scielo.php?script= sci_abstract\&pid=S0034-

98872018000900978\&lng=en\&nrm=iso\&t lng=en

7. Fang X, Zuo J, Zhou J, Cai J, Chen C, Xiang E, et al. Childhood obesity leads to adult type 2 diabetes and coronary artery diseases. Medicine (Baltimore) [Internet]. [citado 12 de diciembre de 2020] 2019;98(32). Disponible en: https://www.ncbi.nlm.nih.gov/pmc/articl es/PMC6708873/

8. Souki Rincon A, Cano-Ponce C, GarcíaCamacho D, Mengual E, González C, Torres D, et al. Variaciones por Edad y Sexo en el HOMAIR, en los niveles de Insulina y Glucosa séricas en niños y adolescentes de Maracaibo-Estado Zulia. Arch Venez Farmacol Ter [Internet]. [citado 16 de diciembre de 2020]; 2007; 26(2):135$1341 . \quad$ Disponible en: http://ve.scielo.org/scielo.php?script=sci_ abstract\&pid=S0798-

02642007000200012\&lng=es\&nrm=iso\&t lng=es

9. Martínez García JJ, Rojas Pérez GG, León Sicairos NM. Prevalencia de resistencia a la insulina y síndrome metabólico en niños obesos que acuden a la Clínica de Obesidad del Hospital Pediátrico de Sinaloa. Pediatría México [Internet]. [citado 16 de diciembre de 2020]; 2010;12(1):18-22. Disponible en: https://www.medigraphic.com/cgi$\mathrm{bin} / \mathrm{new} /$ resumen.cgi?IDARTICULO $=2755$ 6

10. Pajuelo Ramírez J, Bernui Leo I, Sánchez González J, Arbañil Huamán $\mathrm{H}$, Miranda Cuadros M, Cochachin Henostroza 0 , et al. Obesidad, resistencia a la insulina y diabetes mellitus tipo 2 en adolescentes. An Fac Med [Internet]. [citado 19 de diciembre de 2020]; 2018; 79(3):200-5. Disponible en: http://www.scielo.org.pe/scielo.php?scrip $\mathrm{t}=$ sci_abstract\&pid=S1025-
$55832018000300002 \& \operatorname{lng}=\mathrm{es} \& \mathrm{nrm}=\mathrm{iso} \& \mathrm{t}$ lng=es

11. Calderín Bouza RO, Prieto Valdés $M$, Cabrera Rode E. Síndrome de insulinorresistencia en niños $y$ adolescentes. Rev Cuba Endocrinol [Internet]. [citado 12 de diciembre de 2020] 2007; 18(2):0-0. Disponible en: http://scielo.sld.cu/scielo.php?script=sci_a bstract\&pid=S1561-

$29532007000200007 \& \operatorname{lng}=e s \& n r m=i s o \& t$ lng=es

12. Guerra Cabrera C, Vila Díaz J, Apolinaire Pennini J, Cabrera Romero A, Santana Carballosa I, Almaguer Sabina P. Factores de riesgo asociados a sobrepeso y obesidad en adolescentes. MediSur [Internet]. 2009 [citado 12 de diciembre de 2020];7(2):25-34. Disponible en: http://scielo.sld.cu/scielo.php?script=sci_a bstract\&pid=S1727-

897X2009000200004\&lng=es\&nrm=iso\&t lng $=\mathrm{es}$

13. Carrasco F, Galgani JE, Reyes M. Síndrome de resistencia a la insulina. Estudio y manejo. Rev Médica Clínica Las Condes [Internet]. 2013 [citado 12 de diciembre de 2020];24(5):827-37. Disponible en: http://www.sciencedirect.com/science/ar ticle/pii/S071686401370230X

14. Carrillo RM, Miranda J, Gilman RH, Checkley W, Smeeth L, Bernabe-Ortiz A, et al. The HOMA-IR Performance to Identify New Diabetes Cases by Degree of Urbanization and Altitude in Peru: The CRONICAS Cohort Study. J Diabetes Res [Internet]. 2018 [citado 12 de diciembre de 2020]; 2018. Disponible en: https://www.ncbi.nlm.nih.gov/pmc/articl es/PMC6311843/

15. Horáková D, Štěpánek L, Janout V, Janoutová J, Pastucha D, Kollárová $H$, et al. Optimal Homeostasis Model Assessment of Insulin Resistance (HOMA-IR) Cut-Offs: A Cross-Sectional Study in the Czech 
Population. Medicina (Mex) [Internet]. 2019 [citado 12 de diciembre de 2020];55(5). Disponible en: https://www.ncbi.nlm.nih.gov/pmc/articl es/PMC6571793/

16. Lentferink YE, Elst MAJ, Knibbe CAJ, van der Vorst MMJ. Predictors of Insulin Resistance in Children versus Adolescents with Obesity. J Obes [Internet]. 2017 [citado 12 de diciembre de 2020];2017. Disponible en: https://www.ncbi.nlm.nih.gov/pmc/articl es/PMC5742469/

17. Lee $\mathrm{CH}$, Shih AZL, Woo YC, Fong CHY, Leung OY, Janus E, et al. Optimal Cut-Offs of Homeostasis Model Assessment of Insulin Resistance (HOMA-IR) to Identify Dysglycemia and Type 2 Diabetes Mellitus: A 15-Year Prospective Study in Chinese. PLoS ONE [Internet]. 2016 [citado 12 de diciembre de 2020];11(9). Disponible en: https://www.ncbi.nlm.nih.gov/pmc/articl es/PMC5033570/

18. Mirzaalian $Y$, Nourian $M$, Gholamalizadeh M, Doaei S, Hatami M, Hassanzadeh A, et al. The association of quantitative insulin sensitivity indices (HOMA-IR and QUICKI) with anthropometric and cardiometabolic indicators in adolescents. Arch Med Sci Atheroscler Dis [Internet]. 2019 [citado 12 de diciembre de 2020];4:e32-7. Disponible en:

https://www.ncbi.nlm.nih.gov/pmc/articl es/PMC6549040/

19. Morimoto A, Tatsumi $Y$, Soyano F, Miyamatsu N, Sonoda N, Godai K, et al. Increase in Homeostasis Model Assessment of Insulin Resistance (HOMAIR) Had a Strong Impact on the Development of Type 2 Diabetes in Japanese Individuals with Impaired Insulin Secretion: The Saku Study. PLoS ONE [Internet]. [citado 12 de diciembre de 2020]; 2014; 9(8). Disponible en: https://www.ncbi.nlm.nih.gov/pmc/articl es/PMC4148342/
20. Angulo N, Barbella de Szarvas $S$, Mathison Y, Hadad E, González D, Hernández A, et. al. Diagnóstico de resistencia a la insulina por métodos indirectos en escolares obesos. Investig Clínica [Internet]. [citado 12 de diciembre de 2020] 2013;54(2):149-60. Disponible en:

http://ve.scielo.org/scielo.php?script=sci_ abstract\&pid=S0535-

$51332013000200004 \& \operatorname{lng}=e s \& n r m=i s o \& t$ lng=es

21. Cambizaca Mora $G$ del $P$, Castañeda Abascal Ramos I, Sanabria G, Morocho Yaguana LA. Factores que predisponen al sobrepeso y obesidad en estudiantes de colegios fiscales del Cantón Loja-Ecuador. Rev Habanera Cienc Médicas [Internet]. [citado 13 de diciembre de 2020] 2016;15(2):163-76. Disponible en: http://scielo.sld.cu/scielo.php?script=sci_a bstract\&pid=S1729-

519X2016000200004\&lng=es\&nrm=iso\&t $\operatorname{lng}=\mathrm{pt}$

22. Ramos Padilla $P$, Carpio Arias $T$, Delgado López V, Villavicencio Barriga V. Sobrepeso y obesidad en escolares y adolescentes del área urbana de la ciudad de Riobamba, Ecuador. Rev Esp Nutr Humana Dietética [Internet]. [Citado 13 de diciembre de 2020]; 2015;19(1):21-7. Disponible en: http://scielo.isciii.es/scielo.php?script=sci _abstract\&pid=S2174-

$51452015000100004 \& \operatorname{lng}=\mathrm{es} \& \mathrm{nrm}=\mathrm{iso} \& \mathrm{t}$ lng=es

23. Bayas R, Santamaría MV, Buitrón LR. Prevalencia de Síndrome Metabólico, Índice de Masa Corporal y Resistencia Insulínica en estudiantes de la Universidad Tecnológica Equinoccial, Quito - Ecuador 2011. Tsafiqui - Rev Científica En Cienc Soc [Internet]. [citado 12 de diciembre de 2020]; 2014;(6):49-61. Disponible en: https://revistas.ute.edu.ec/index.php/tsafi qui/article/view/231 
24. Jiménez Cabrera F, Estrada C, Carbo L, Palma L. La hipertrigliceridemia como marcador temprano de resistencia a la insulina en obesidad infanto-juvenil. Rev Cuba Pediatría [Internet]. [citado 13 de diciembre de 2020] 2018;90(3). Disponible en: http://www.revpediatria.sld.cu/index.php /ped/article/view/485

25. Hutton B, Catalá-López F, Moher D. La extensión de la declaración PRISMA para revisiones sistemáticas que incorporan metaanálisis en red: PRISMA-NMA. Med Clínica [Internet]. [citado 12 de diciembre de 2020] 2016;147(6):262-6. Disponible en: http://www.elsevier.es/es-revistamedicina-clinica-2-articulo-la-extensiondeclaracion-prisma-revisionesS0025775316001512

26. Acosta B A, Escalona O M, Maiz G A, Pollak C F, Leighton P F. Determinación del índice de resistencia insulínica mediante HOMA en una población de la Región Metropolitana de Chile. Rev Médica Chile [Internet]. [citado 12 de diciembre de 2020]; 2002;130(11):1227-31. Disponible en:

https://scielo.conicyt.cl/scielo.php?script= sci_abstract\&pid=S0034-

98872002001100004\&lng=es\&nrm=iso\&t lng=es

27. Shashaj B, Luciano R, Contoli B, Morino GS, Spreghini MR, Rustico C, et al. Reference ranges of HOMA-IR in normalweight and obese young Caucasians. Acta Diabetol [Internet]. 2016;53(2):251-60. Disponible en: https://www-scopuscom.vpn.ucacue.edu.ec/record/display.uri ?eid=2-s2.0-

84930903028\&origin=resultslist\&sort=plf -f\&src=s\&st1=\&st2=\&sid=bf0b481adb4f 26ae859a13bddc44dc50\&sot=b\&sdt=b\&sl $=87 \& \mathrm{~s}=$ TITLE-ABS-

KEY+\%28Reference+ranges+of +HOMAIR+in+normalweight+and+obese+young+Caucasians $\% 2$ 9 \&relpos $=0 \&$ citeCnt $=60 \&$ searchTerm $=$
28. Kurtoğlu S, Hatipoğlu N, Mazıcıoğlu M, Kendirici M, Keskin M, Kondolot M. Insulin Resistance in Obese Children and Adolescents: HOMA-IR Cut-Off Levels in the Prepubertal and Pubertal Periods. I Clin Res Pediatr Endocrinol [Internet]. [citado 28 de febrero de 2021]; 2010; 2(3):100-6. Disponible en: https://www.ncbi.nlm.nih.gov/pmc/articl es/PMC3005684/

29. Atabek M, Pirgon O. Assessment of insulin sensitivity from measurements in fasting state and during an oral glucose tolerance test in obese children. J Pediatr Endocrinol Metab [Internet]. 2007;20(2):187-95. Disponible en: https://www-scopus-

com.vpn.ucacue.edu.ec/record/display.uri ?eid=2-s2.0-

$34247126562 \&$ origin=resultslist $\&$ sort $=$ plf

$\mathrm{f} \& \mathrm{src}=\mathrm{s} \& \mathrm{st} 1=\& \mathrm{st} 2=\& \mathrm{sid}=9 \mathrm{aa} 47 \mathrm{ddeb} 8 \mathrm{ea} 34$ d127393d017f9d 908e\&sot=b\&sdt=b\&sl=1 44\&s=TITLE-ABS-

KEY+\%28Assessment+of+Insulin+Sensitiv ity+from+Measurements+in+Fasting+State + and+During+an+Oral+Glucose+Tolerance + Test + in+Obese+Children\%29\&relpos $=2 \&$ citeCnt $=62$ \&searchTerm $=$

30. Singh $Y$, Garg $M$, Tandon N, Marwaha RK. A Study of Insulin Resistance by HOMAIR and its Cut-off Value to Identify Metabolic Syndrome in Urban Indian Adolescents. J Clin Res Pediatr Endocrinol [Internet]. [citado 28 de febrero de 2021] 2013;5(4):245-51. Disponible en: https://www.ncbi.nlm.nih.gov/pmc/articles /PMC3890224/

31. Madeira I, Carvalho CNM, Gazolla FM, de Matos HJ, Borges MA, Bordallo MAN. Cut-off point for Homeostatic Model Assessment for Insulin Resistance (HOMA-IR) index established from Receiver Operating Characteristic (ROC) curve in the detection of metabolic syndrome in overweight prepubertal children. Arq Bras Endocrinol Metabol [Internet]. 2008;52(9):1466-73. 
Disponible en: https://pubmed.ncbi.nlm.nih.gov/19197455 /

32. Burrows R, Correa-Burrows $\mathrm{P}$, Reyes $\mathrm{M}$, Blanco E, Albala C, Gahagan S. Healthy Chilean Adolescents with HOMA-IR $\geq 2.6$ Have Increased Cardiometabolic Risk: Association with Genetic, Biological, and Environmental Factors. J Diabetes Res [Internet]. 2015 [citado 28 de febrero de 2021] 2015. Disponible en: https://www.ncbi.nlm.nih.gov/pmc/articles /PMC4530255/

33. Burrows R, Leiva LB, Weisstaub G, Lera LM, Albala CB, Blanco E, et al. High HOMA-IR, adjusted for puberty, relates to the metabolic syndrome in overweight and obese Chilean youths. Pediatr Diabetes [Internet]. 2011;12(3 Pt 2):212-8. Disponible en: https://pubmed.ncbi.nlm.nih.gov/21426454 /

34. Cândido A, Geloneze B, Calixto A, Vasques ACJ, Freitas RN, Freitas SN, et al. Adiponectin, HOMA-Adiponectin, HOMA-IR in Children and Adolescents: Ouro Preto Study. Indian J Pediatr [Internet]. 2020; Disponible en: scopuscom.vpn.ucacue.edu.ec/record/display.uri?e $\mathrm{id}=2$-s2.0-

85091188720\&origin=resultslist\&sort=plf$\mathrm{f} \& \mathrm{src}=\mathrm{s} \& \mathrm{st} 1=\& \mathrm{st} 2=\& \mathrm{sid}=0 \mathrm{ac} 8708 \mathrm{a} 0 \mathrm{f} 0 \mathrm{eb} 2 \mathrm{fa}$ 617ee13098acaa8d\&sot=b\&sdt=b\&sl=100\& $\mathrm{s}=$ TITLE-ABS-

KEY+\%28Adiponectin\%2c+HOMA

35. Brar $\mathrm{P}$, Mengwall L, Franklin $\mathrm{BH}$, Fierman AH. Screening obese children and adolescents for prediabetes and/or type 2 diabetes in pediatric practices: a validation study. Clin Pediatr (Phila) [Internet]. 2014;53(8):771-6. Disponible en: https://pubmed.ncbi.nlm.nih.gov/246718 74/

36. Lee JM, Okumura MJ, Davis MM, Herman WH, Gurney JG. Prevalence and determinants of insulin resistance among U.S. adolescents: a population-based study.
Diabetes Care [Internet]. 2006;29(11):2427-32. Disponible en: file://C:/Users/Usuario/Zotero/storage/ 8WGZWWJY/Lee\%20et\%20al.\%20$\% 202006 \% 20$ -

$\% 20$ Prevalence $\% 20$ and $\% 20$ determinants \%20of\%20insulin\%20resistance\%20.pdf

37. Ferrannini E, Natali A, Bell P, CavalloPerin $\mathrm{P}$, Lalic N, Mingrone G. Insulin resistance and hypersecretion in obesity. European Group for the Study of Insulin Resistance (EGIR). J Clin Invest [Internet]. 1997;100(5):1166-73. Disponible en: https://pubmed.ncbi.nlm.nih.gov/930392 $3 /$

38. Rocco ER, Mory DB, Bergamin CS, Valente F, Miranda VL, Calegare BFA, et al. Optimal cutoff points for body mass index, waist circumference and HOMA-IR to identify a cluster of cardiometabolic abnormalities in normal glucose-tolerant Brazilian children and adolescents. Arq Bras Endocrinol Metabol [Internet]. 2011;55(8):638-45. Disponible en: https://pubmed.ncbi.nlm.nih.gov/222184 48/

39. Siqueira M, Oliveira JS, Leal VS, da Lima NMS, Costa EC, de Aquino NB, et al. Identification of cutoff points for Homeostatic Model Assessment for Insulin Resistance index in adolescents: systematic review. Rev Paul Pediatr [Internet]. [citado 8 de marzo de 2021] 2016;34(2):234-42. Disponible en: https://www.ncbi.nlm.nih.gov/pmc/articl es/PMC4917276/

40. Cerda J, Cifuentes L. Uso de curvas ROC en investigación clínica: Aspectos teóricoprácticos. Rev Chil Infectol [Internet]. [citado 8 de marzo de 2021] 2012;29(2):138-41. Disponible en: https://scielo.conicyt.cl/scielo.php?script= sci_abstract\&pid=S0716-

$10182012000200003 \& \operatorname{lng}=e s \& n r m=i s o \& t$ lng=es 
41. Conwell L, Trost SG, Brown WJ, Batch JA. Indexes of insulin resistance and secretion in obese children and adolescents: a validation study. Diabetes Care [Internet]. 2004;27(2):314-9. Disponible https://pubmed.ncbi.nlm.nih.gov/147472 06/

42. Keskin M, Kurtoglu S, Kendirci M, Atabek ME, Yazici C. Homeostasis Model Assessment Is More Reliable Than the Fasting Glucose/Insulin Ratio and Quantitative Insulin Sensitivity Check Index for Assessing Insulin Resistance
Among Obese Children and Adolescents. Pediatrics [Internet]. [citado 7 de marzo de 2021]; 2005;115(4):e500-3. Disponible en: https://pediatrics.aappublications.org/con tent/115/4/e500

Conflicto de intereses. La investigación se enmarcó en los estándares éticos, en pro de mejorar los procesos institucionales.

No fue requerida ayuda financiera de ninguna institución gubernamental o no gubernamental.

Los autores declaran no tener ningún conflicto de intereses. 